\title{
Well-known and less well-known anatomical structures of the head, neck, and trunk: imaging and surgical applications
}

\author{
Bruno Grignon $^{1} \cdot$ Fabrice Duparc ${ }^{2}$ (1)
}

Published online: 21 July 2021

(c) The Author(s), under exclusive licence to Springer-Verlag France SAS, part of Springer Nature 2021

First described in 2012, the glymphatic system is defined as a network of perivascular spaces promoting movement of the cerebrospinal fluid into the brain and clearance of metabolic waste. By means of both magnetic resonance imaging (MRI) and dissection, a study of the glymphatic pathway of the optic nerve is detailed in this issue.

Arachnoid granulations are very well-known normal structures that may sometimes have an unusual ectatic appearance requiring differentiation from various pathological entities such as sinus thromboses, meningiomas, cavernous hemangiomas and meningoceles in particular. A review of these structures has been performed in 102 subjects using MRI, highlighting the interest of thin-sliced contrast MRI in the field on one hand, and the most frequent location of these bulging arachnoid granulations in the transverse sinus on the other hand.

The calvarial diploic channels are distinct pathways (within the diploe) that can be involved in various epidural hematomas, sometimes complicating surgical procedures. A better knowledge of their three-dimensional structure may be provided by MRI using MIP (maximum intensity projection) reformations, as was demonstrated by a study carried out in 77 patients.

Imaging of the trochlear nerve, the smallest in diameter and the longest in intracranial course of the twelve cranial nerves, may be of great importance since it can be affected by various disorders, among others in the trochlear cistern of

Fabrice Duparc

fabrice.duparc@univ-rouen.fr

Bruno Grignon

b.grignon@chu-nancy.fr

1 Department of Anatomy, Faculty of Medicine, University of Lorraine, Avenue de la Forêt de Haye, 54505 Vandœuvre-Lès-Nancy, France

2 Department of Anatomy, Faculty of Medicine-Pharmacy, University of Rouen-Normandy, 22 Boulevard Gambetta, 76183 Rouen, France the cavernous sinus. A study of 43 subjects proposed in this number confirmed that MRI stands as a technique of choice for imaging this segment.

Thin-sliced contrast MRI may also be of particular interest for exploring the inferior sagittal sinus, a well-known minor dural sinus of the lower border of the falx cerebri, sometimes involved in sinus thromboses, venous aneurysm dural arteriovenous fistulas, or arteriovenous malformations, as suggested by the investigation of 77 patients with intact cerebral hemispheres and covering meninges.

The subcochlear canaliculus is an anatomical cavity, originating in the space between the fustis and the funiculus and connecting the round window area to the petrous apex, the pneumatization of which having important surgical implications in particular in cholesteatoma surgery and cochlear implantation surgery. The study of 270 petrous bone computed tomography scans presented in this number provides a significant contribution in the field.

An important anatomical landmark that is sometimes removed in certain surgical interventions, the anterior clinoid process presents complex anatomical relationship with surrounding neurovascular structures. These structures have been systematically and carefully reviewed by means of a literature review, and extradural and intradural clinoidectomy techniques in six dry bone heads and ten injected cadaverous specimens.

Rare variations of encephalic vessels may sometimes be of great interest for various surgical procedures. An example is given in this issue through a combined persistent primitive olfactory artery and azygos pericallosal artery demonstrated and carefully documented by computed tomography angiography with three-dimensional volume rendering technique.

The complexity of distinction of anastomoses between the ophthalmic artery and the middle meningeal arteries versus accessory middle meningeal arteries is also discussed and illustrated.

In the neck, the local variations of the sympathetic ganglia according to vertebral levels may be of crucial 
importance during various surgical procedures. Investigating these localizations by designating the carotid artery as the standard landmark at the center was the aim of the study of 231 subjects using MRI of both 1.5 and $3 \mathrm{~T}$ devices, the results of which are presented and commented in this issue.

Some aspects of the anatomical basis of three transcutaneous injection laryngoplasty techniques using three-dimensional reconstructed computed tomography have also been investigated.

Anatomical variations of the infrahyoid muscles may have sometimes practical applications for surgical purposes, as is the case of an unreported variant of cleidohyoid muscle presented in this number, that could be named the "sternocleidohyoid" muscle.

In the field of the upper abdominal surgery, the anatomical variations of the splenic artery may be of paramount importance. A systematic review of literature gathering 3132 specimens is presented and illustrated.

Vagus nerve injuries during gastroesophageal surgery may cause significant symptoms. The high anatomical variability of this nerve must be well known in order to avoid such complications and has been investigated by a new analytical cross-sectional survey of 67 bodies and discussed in the light of the literature.

The concept of mesopancreas has recently been brought into focus and remains discussed. This concept has been revisited in the light of a study of 33 bodies including gross anatomy and histological observation after transection.

Finally, at the cross-road of anatomy and pathology, hepatic hotspots are surprising images that could be seen on angio-computed tomography in case of superior vena cava syndrome. They are due to a complex variant venous network, which has been very carefully analyzed, documented and illustrated in this issue.

Wishing you both a pleasant summer and an enjoyable reading,

Publisher's Note Springer Nature remains neutral with regard to jurisdictional claims in published maps and institutional affiliations. 